\title{
A Novel Fuzzy Logic Guided Method for Automatic gEUD-based Inverse Treatment Planning
}

\author{
Caiping Guo ${ }^{1}$, Linhua Zhang ${ }^{2}$, Jiahui Peng ${ }^{1}$ \\ ${ }^{1}$ Electronic Engineering Department, Taiyuan Institute of Technology, Taiyuan 030008, China \\ ${ }^{2}$ Computer Engineering Department, Taiyuan Institute of Technology, Taiyuan 030008, China
}

Received: October 27, 2020. Revised: May 19, 2021. Accepted: June 2, 2021. Published: June 7, 2021.

\begin{abstract}
Generalized equivalent uniform dose (gEUD) -based hybrid objective functions are widely used in intensity modulated radiotherapy (IMRT). To improve its efficiency, a novel fuzzy logic guided inverse planning method was developed for the automatic parameters optimization of the gEUD-based radiotherapy optimization. Simple inference rules were formulated according to the knowledge of the treatment planner. Then they automatically and iteratively guide the parameters modification according to the percentage of deviation between the current dose and the prescribed dose. weighting factors and prescribed dose were automatically adjusted by developed fuzzy inference system (FIS). The performance of the FIS was tested on ten prostate cancer cases. Experimental results indicate that proposed automatic method can yield comparable or better plans than manual method. The fuzzy logic guided automatic inverse planning method of parameters optimization can significantly improve the efficiency of the method of manually adjusting parameters, and contributes to the development of fully automated planning.
\end{abstract}

Keywords-Weighting factors, Intensity-modulated radiation therapy, Prescribed dose, Automatic planning, fuzzy logic.

\section{INTRODUCTION}

Intensity modulated radiotherapy (IMRT) inverse planning has been widely used to spare the dose to organs at risk (OARs) and conform the dose to planning target volume (PTV). In clinical inverse planning, the planner will have to define several parameters, such as weighting factors and bounds, to attain a desired plan by lengthy trial-and-error procedure. As these parameters are iteratively changed based on planner's reasoning and experience until a proper combination of parameters and an acceptable plan are reached, it could be accomplished by fuzzy logic belonging to artificial intelligence (AI) ${ }^{[1]}$.

To date, several researchers have introduced fuzzy logic into physical optimization of radiotherapy. LI and Yin ${ }^{[2]}$ applied fuzzy logic into a treatment planning to optimize weighting factors for normal tissue (NT). Yan et al. ${ }^{[3]}$ used a fuzzy inference technique to optimize the weighting factors. Yan et al. ${ }^{[4]}$, Stieler et al. ${ }^{[5]}$, and Dias et al. ${ }^{[6]}$ proposed fuzzy logic guided parameters optimization in physical radiation, including weighting factors and prescribed dose. With the advantages ${ }^{[7]}$ and developing trend of biological optimization or hybrid physical-biological optimization, it is necessary to extend this approach to parameter optimization for them. The biological criteria applied to treatment planning include tumor control probability (TCP), normal tissue complication probability (NTCP), and generalized equivalent uniform dose (gEUD). As the uncertainty associated with them, model based on TCP or NTCP has not been widely used in inverse system ${ }^{[8]}$. However, gEUD guided inverse planning has been investigated ${ }^{[9-18]}$ and clinically applied in Varian system [19] because of its advantages ${ }^{[10]}$. To improve the efficiency of gEUD-based inverse planning, it is desirable to have an automatic, or a more effective approach.

In our study, we reported a fuzzy inference guided automatic method to optimize the combination of parameters for the gEUD-based treatment planning. The propose methodology was tested on ten prostate clinical cases. In the following sections, the method of fuzzy inference system guided gEUD-based optimization is described in details in Section II. Then, the feasibility and effectiveness of the proposed automatic method are demonstrated by comparing with the manual method in Section III. Section IV concludes the main findings and points out the future research directions.

\section{MATERIALS AND METHODS}

\section{A. Objective Function}

Some researchers ${ }^{[12,16]}$ have demonstrated the superiority of gEUD-based hybrid optimization compared with gEUD-based biological optimization. Based on their study, the subjective function we used in our study can be formulated as

$$
f(D(x))=\xi_{1} f_{g E U D}\left(D(x)^{\text {bladder }}\right)+\xi_{2} f_{g E U D}\left(D(x)^{\text {rectum }}\right)+\xi_{3} f_{\text {min }}\left(D(x)^{P T V}\right)+\xi_{4} f_{\text {max }}\left(D(x)^{P T V}\right)
$$

Where a minimum dose sub-score of $f_{\min }\left(\boldsymbol{D}(\boldsymbol{x})^{P T V}\right)$ and a maximum dose sub-score of $f_{\max }\left(\boldsymbol{D}(\boldsymbol{x})^{P T V}\right.$ were used to 
guarantee the therapeutic dose delivered to the PTV (planning target volume), along with two gEUD-based sub-scores of $f_{\text {gEUD }}\left(\boldsymbol{D}(\boldsymbol{x})^{\text {bladder }}\right)$ and $f_{\text {gEUD }}\left(\boldsymbol{D}(\boldsymbol{x})^{\text {rectum }}\right)$ to minimize the dose delivered to the bladder and rectum walls, respectively. $\boldsymbol{D}(\boldsymbol{x})=\boldsymbol{W} \boldsymbol{x}$ is the dose distribution vector, $\boldsymbol{x}$ is the beamlet weights, $\boldsymbol{W}$ is the dose deposition matrix computed using the CERR pencil beam algorithm (QIB) with corrections for heterogeneities ${ }^{[20]}$. The $f_{\min }(\boldsymbol{D}(\boldsymbol{x}))$ and $f_{\max }(\boldsymbol{D}(\boldsymbol{x}))$ was proposed by Wu and Mohan ${ }^{[21]}$, and the gEUD sub-score is expressed as ${ }^{[14,18]}$

$$
f_{g E U D}(\boldsymbol{D}(\boldsymbol{x}))=\max \left(0, g E U D(\boldsymbol{D}(\boldsymbol{x}))-g E U D_{0}\right)
$$

Here, $g E U D(D(x))$ is the current dose, and $g E U D_{0}$ is the prescription dose. $g E U D(\boldsymbol{D}(\boldsymbol{x}))$ is given by Equation (3) ${ }^{[22]}$

$$
\operatorname{gEUD}(\boldsymbol{D}(\boldsymbol{x}))=\left(\frac{1}{N} \sum_{j=1}^{N}\left(\boldsymbol{w}_{j} \boldsymbol{x}\right)^{a}\right)^{l / a}
$$

$\mathrm{N}$ is the number of voxels in the optimized anatomical structure of interest, a is the tissue-specific parameter representing the dose-volume effect, $\boldsymbol{w}_{j}$ corresponding to voxel $\mathrm{j}$, is the jth row of the dose deposition matrix.

\section{B. Fuzzy Inference Mechanism}

In 1965 , to characterize non-probabilistic uncertainties, Zadeh proposed an approach called fuzzy-sets ${ }^{[23-26]}$.To simulating the reasoning process generally performed by a human, the fuzzy inference system (FIS) was applied in some industrial applications [27-29]. Some relevant studies can be found in [30] and [31] A Mamdani-type FIS includes three components: fuzzifier, inference engine, and defuzzifier shown in Figure $1^{[5]}$.

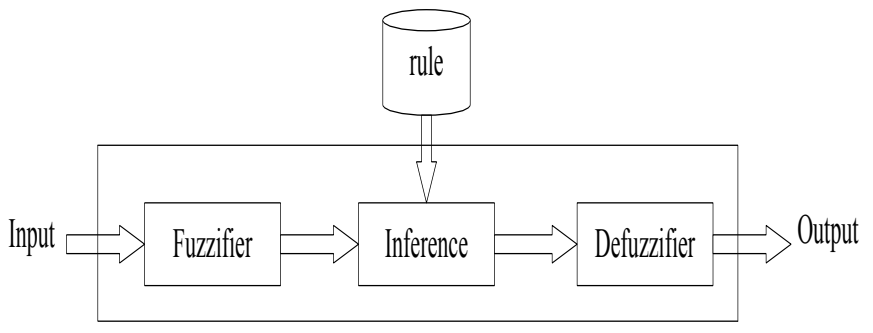

Figure 1. The illustration of a fuzzy inference system

The inputs are processed by the fuzzifier based on the membership function for the inputs. The inference part processes the result values and calculates the consequences according to the rule. Then the defuzzifier converts the consequence to the final outputs. The constituents of the rules mainly influence the behavior of a FIS. Input and output variables are portioned into different spaces according to fuzzy sets. Then, create an appropriate function to map the input / output spaces to the real numbers called membership values.

Two fuzzy inference systems were needed for the automatic method. One is specified for the modification of the weighting factor, the other is specified for the modification of the prescribed dose. The two kinds of parameters are optimized alternately. It should be noted that when the prescribed doses are changed, the weighting factors take their initial values, and the algorithm processes by changing them again. Our first approach to this problem considered changing the prescribed doses directly after the process of weighting factors adjustment, but it is more time consuming. Taking the initial weighting factors when prescribed doses are changed proved to be a better choice.

In our work, we used triangular and trapezoidal membership functions. Figure 2 and Figure 3 illustrate the membership functions considered for determining the change in the weighting factors (WF) and prescribed dose (PD).

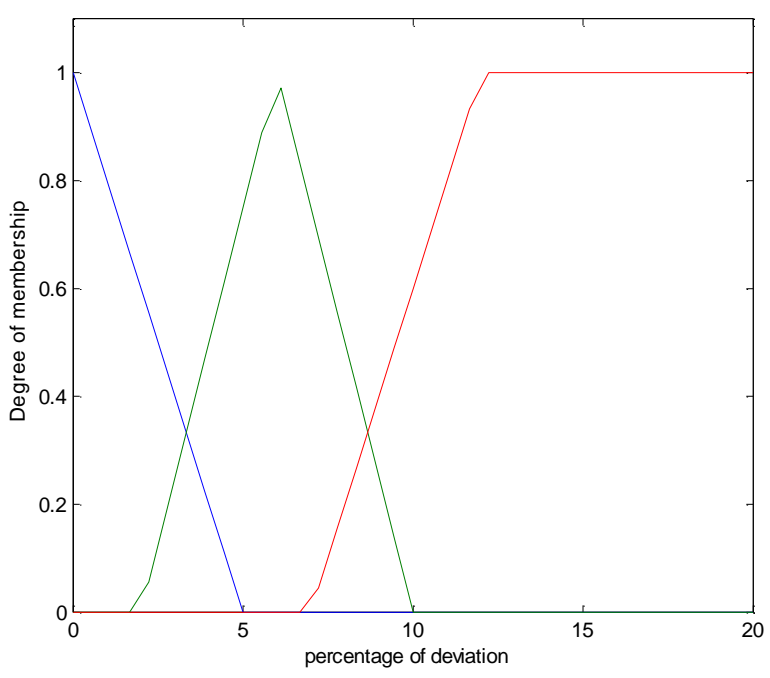

a)The input of Prescribed dose for PTV

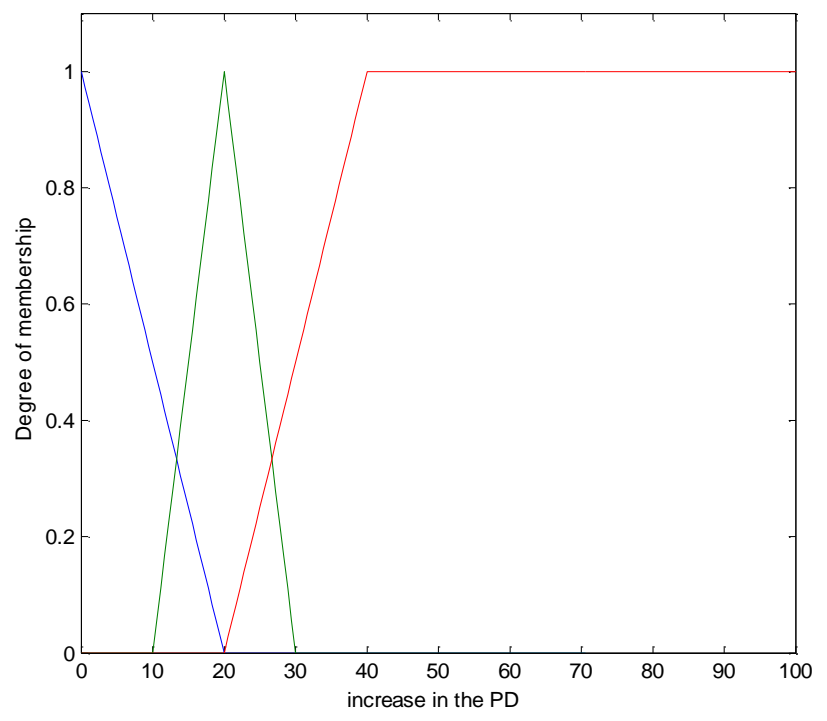

b)The output of Prescribed dose for PTV 


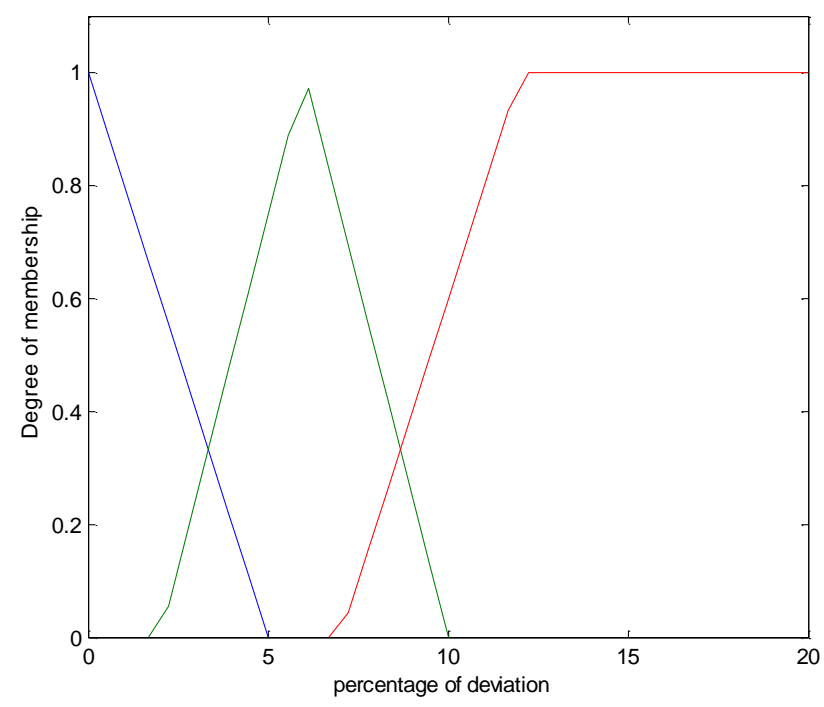

c)The input of Weighting factor for PTV

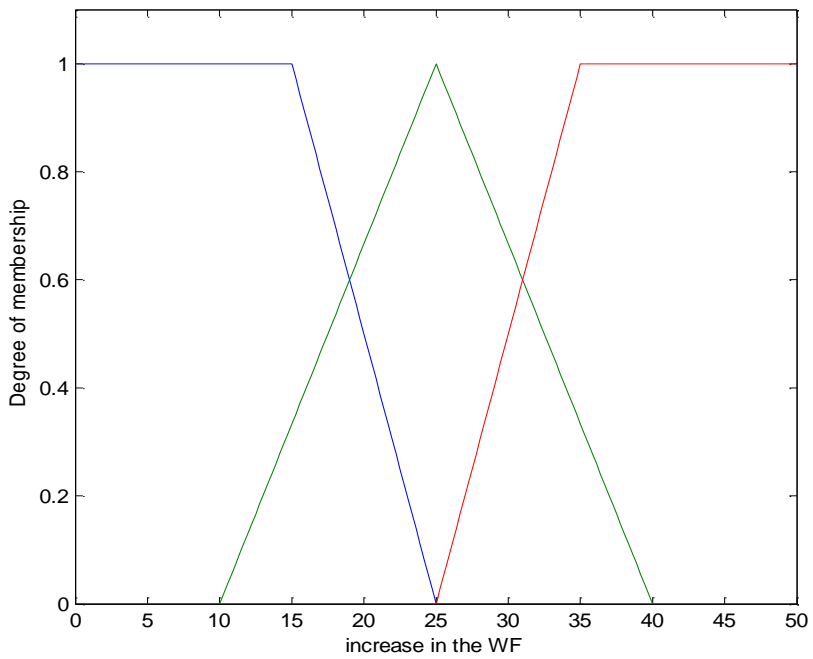

d)The output of Weighting factor for PTV

Figure 2. Membership functions PTV for FIS

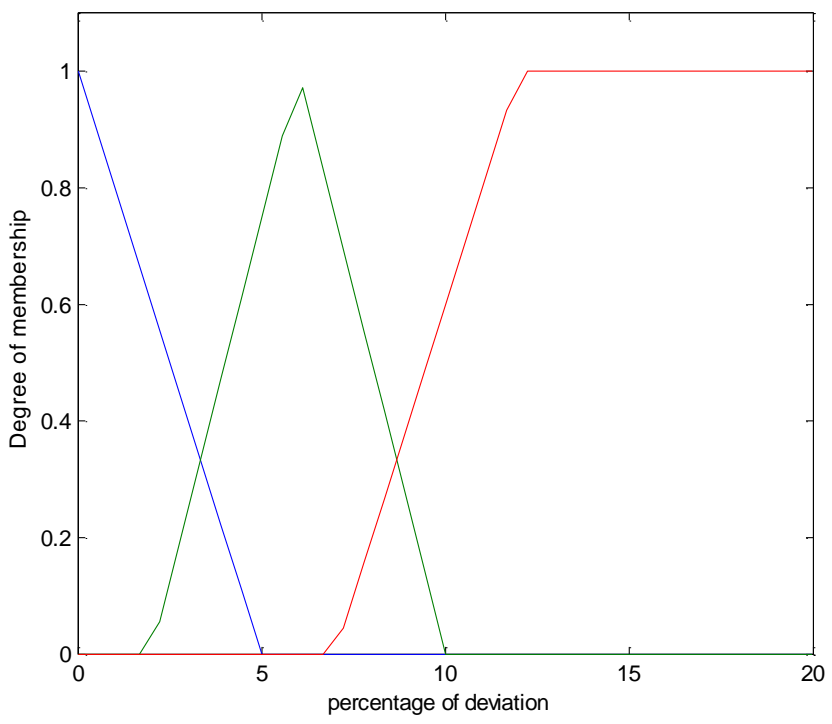

a)The input of Prescribed dose for OAR

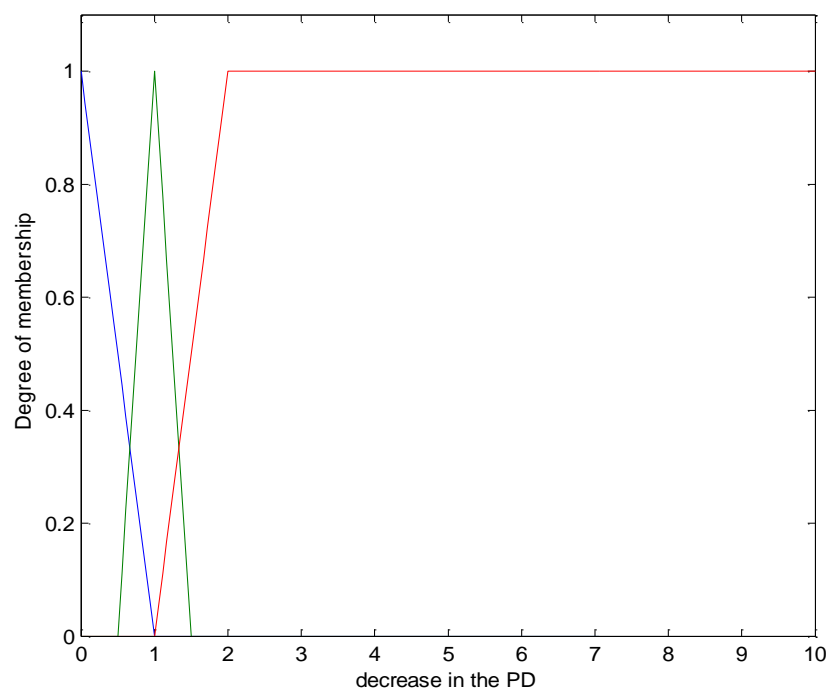

b)The output of Prescribed dose for OAR

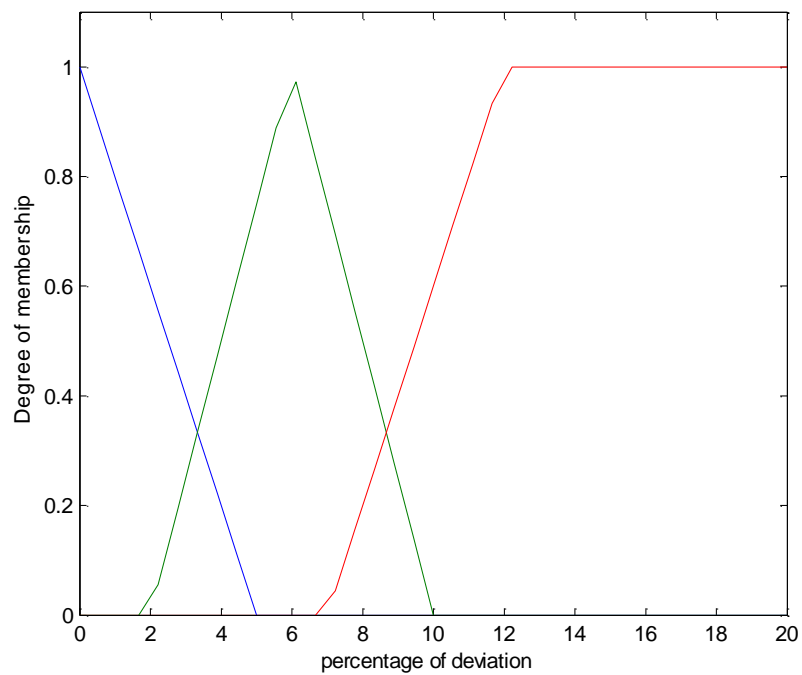

c)The input of Weighting factor for OAR

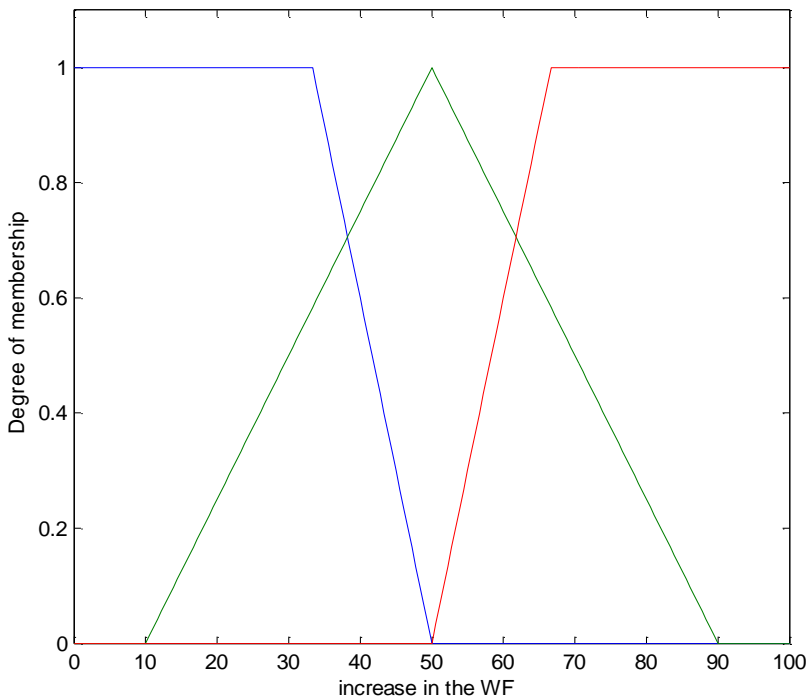

d)The output of Weighting factor for OAR

Figure 3. Membership functions OAR for FIS 
As shown in Figure 2 and Figure 3, for different sub-scores (physical or biological), the output function of FIS are different, because of their inherent differences. The input, ranging from $0 \%$ to $100 \%$, is calculated by the percentage of deviation between the current dose and the prescribed dose,and this is simply a measure of how much the constraint is being violated. The output is given by the change in the PD or WF. Weighting factor and prescribed dose are changed following very simple common-sense rules: if a given dose constraints is being violated, then change the weighting factor or prescribed dose that can contribute to the satisfaction of the constraint. For example, imaging that the current plan with $D_{\min }^{P T V}=73.8 G y$ and $D_{\max }^{P T V}=80 G y$, the algorithm will compute the deviation in percentage $0.27 \%$ and $2.56 \%$, then the increase change of the prescribed $D_{\min }^{P T V}$ and the decrease change of the prescribed $D_{\max }^{P T V}$ are determined according to their FISs. As soon as the PD is updated for every optimized structure, the FMO is solved again. Similar to the one described for PD modification, the weights are modified according to proposed FIS.

A set of fuzzy rules connected the input and output membership functions together. In our work, we applied three fuzzy rules that should be evaluated simultaneously: low deviation implying low change, medium deviation implying medium change, and high deviation implying high change. A set of truncate output fuzzy sets are generated after evaluating all the fuzzy rules, then a single value is calculated according to these sets. This procedure is called defuzzification. Here, the way of the centroid calculation defuzzification is applied.

\section{Optimization Workflow}

Next, we describe the whole algorithm procedure. "IT" represents the total numbers of FMO, "Improve" denotes whether or not the dose coverage of PTV is further improved, and "Admissible" represent whether or not the current plan is acceptable.

Step 1. Determine a set of beam angles. Improve $\leftarrow 0$; IT $\leftarrow 0$.

Step 2. Initialize all the models parameters (prescribed dose, initial beamlet weights, initial weighting factors, et al.).

Step 3. Solve the fluence map optimization (FMO) with the current parameters; IT $\leftarrow$ IT +1 .

Step 4. Dose calculations. Admissible $\leftarrow$ True.

Step 5. For each optimized organs, the following decision is made. If there is a deviation between the current dose metrics and the desired ones for structure $s$, change the weighting factor associated with it according to fuzzy inference syetem. Admissible $\leftarrow$ False, repeat steps 3 to 5 once. If the current dose satisfies the prescribed dose, go to 7 .

Step 6. If Admissible $\leftarrow$ False, then we have as follows. For structure $\mathrm{s}$, change the prescribed dose using the fuzzy inference system. Repeat steps 2 to 5 until an acceptable plan is reached or IT reaches the maximum number of iterations $\mathrm{N}_{\max }$. Step 7. If Admissible $\leftarrow$ True, Improve $\leftarrow 1$, change the desired dosimetry objectives for PTV, trying to improve PTV coverage while ensuring the prescribed OARs constraints. Go to Step3.

The algorithm will be able to calculate a plan that is complying as much as possible with the defined treatment constraints.

Plan evaluation is performed to determine whether the current plan is acceptable. The physician has defined 78Gy for the PTV and wants V $\left(\mathrm{D}_{95 \%}\right)$ to be at least $95 \%$, meanwhile, V (80Gy) to be 0 . For rectum and bladder sparing, the clinical practice guidelines ${ }^{[32]}$ were applied to evaluate them. By alternately changing the PD and WF, the algorithm will direct the search process and will try to find a solution that meets all the prescribed constraints. After an acceptable plan is obtained, the algorithm will try to improve PTV coverage. At this stage, this is realized by changing obtained $D_{\min }$ to $D_{\min }+0.5$ : if the planner has defined an acceptable plan with $D_{\min }$ equal to $74 \mathrm{~Gy}$, then the algorithm will try to guarantee that $D_{\min }$ is equal to or greater than $74.5 \mathrm{~Gy}$. The procedure is repeated until the PTV coverage cannot be further improved.

\section{Patients, Related Parameters, and Assessment Criteria}

The feasibility and performance of the automatic method was tested on 10 cases of prostate cancer. The experiment environment is the same with that used in our previous work [33], and the FMO were solved using gradient-based optimization algorithm (namely, L-BFGS) ${ }^{[34]}$.The study protocols were approved by the Ethics Committee of the North University of China with the approval No.2018006,the written consent forms were signed by the subjects whose Computed Tomography (CT) images were used for this study. For prostate cancer cases, these patients underwent simulation and treatment in the supine position. Target volume and organs at risk (bladder, rectum, and femoral heads) were delineated on CT slices. The rectum and bladder walls were generated with a thickness of $5 \mathrm{~mm}$ from the external manually-delineated rectal and bladder contours, respectively. A "tissue ring", the outside of the area extending the PTV by $5 \mathrm{~cm}$, was defined as the other normal tissue. The target volume included the prostate and excluded the pelvic lymph nodes. The PTV was calculated by adding a $10 \mathrm{~mm}$ margin in all directions except the posterior, where a $5 \mathrm{~mm}$ margin was applied. All plans used the identical configuration of five coplanar $6 \mathrm{MV}$ photon beams, with gantry angles of $36^{\circ}, 100^{\circ}, 180^{\circ}, 260^{\circ}$, and $324^{\circ}$. The prescription doses for each sub-score in Equation (1) are initialized considering the desired prescription defined by the physician. The prescribed $g E U D_{0}$ for rectum and bladder were $60 \mathrm{~Gy}{ }^{[35]}$, and the prescribed dose $D_{\min }$ and $D_{\max }$ were $74 \mathrm{~Gy}$ and $78 \mathrm{~Gy}$, respectively. Volume effect parameter $a=8$ was applied to calculate $g E U D^{[36]}$. The uniform initial weighting factors $(1 / 4$, $1 / 4,1 / 4,1 / 4)$ were used.

All experiments were performed by using an instrument equipped with a 32-bit OS, Windows 7, and an Intel (R) Core (TM) i3-4150 CPU with 4G RAM.

The plan quality was assessed by using the clinical evaluation guidelines ,conformity index (CI) and homogeneity index $(\mathrm{HI})^{[37]}$ respectively defined in Equation (4) and Equation (5).

$$
C I=\frac{V_{\tau, r e f}}{V_{\tau}} \cdot \frac{V_{\tau, r e f}}{V_{r e f}},
$$


where $V_{\tau}$ is the volume of the PTV, $V_{\tau, \text { ref }}$ is the target volume that receives a dose greater than or equal to the reference (prescribed)dose, and $V_{\text {ref }}$ is the total volume that receives a dose greater than or equal to the reference dose.

$$
H I=\frac{D_{5 \%}}{D_{95 \%}},
$$

where $D_{5 \%}$ and $D_{95 \%}$ correspond to the minimum doses delivered to the hottest $5 \%$ and $95 \%$ of the PTV, respectively.

\section{RESULTS}

For all patients, admissible plans can be found applying our proposed automatic method. Table 1 shows the Conformal index (CI) and homogeneity index (HI) of automatic plan for each prostate case It is clearly that the proposed automatic method yields good dose conformity and homogeneity to the PTV.

Table 1. Dose Conformity and Homogeneity to the PTV of the automatic plan for 10 prostate cases.

\begin{tabular}{cccccc}
\hline & \multicolumn{5}{c}{ Case } \\
\cline { 2 - 6 } Index & 1 & 2 & 3 & 4 & 5 \\
\hline CI & 0.93 & 0.91 & 0.88 & 0.92 & 0.90 \\
\hline HI & 1.02 & 1.02 & 1.04 & 1.02 & 1.04 \\
\hline Index & \multicolumn{5}{c}{ Case } \\
\cline { 2 - 6 } & 6 & 7 & 8 & 9 & 10 \\
\hline CI & 0.89 & 0.91 & 0.90 & 0.87 & 0.90 \\
\hline HI & 1.02 & 1.02 & 1.02 & 1.04 & 1.02 \\
\hline
\end{tabular}

Moreover, it was possible to further improve PTV coverage for the majority of cases. Table 2 lists the number of FMO of finding the first admissible plan and the final plan. The meaning of "improve" is consistent with the above definition. The number of FMO corresponding to the first admissible plan shows how many times the process of FMO runs. After obtaining the first acceptable plan, the algorithm tries to improve PTV coverage. The final plan corresponds to the last improved plan. The differences of the numbers of FMO for each patient mainly contribute the complexity of the patients anatomy.

Table 2. Numbers of FMO for each patient.

\begin{tabular}{lccccc}
\hline Number of FMO & \multicolumn{5}{c}{ Patients } \\
\cline { 2 - 6 } & 1 & 2 & 3 & 4 & 5 \\
\hline First admissible plan & 2 & 4 & 4 & 8 & 6 \\
Final plan & 4 & 8 & 8 & 8 & 6 \\
Improve & 1 & 1 & 1 & 0 & 0 \\
Number of FMO & & \multicolumn{4}{c}{ Patients } \\
First admissible plan & 6 & 7 & 8 & 9 & 10 \\
Final plan & 2 & 2 & 2 & 2 & 4 \\
Improve & 7 & 7 & 8 & 2 & 10 \\
\hline
\end{tabular}

Figure 4 shows the comparison result between the first admissible plan and the final improved plan for patient 1 . It can be seen that with the improvement of the PTV coverage, the dose to OARs increased due to the inherent contradiction of radiotherapy.

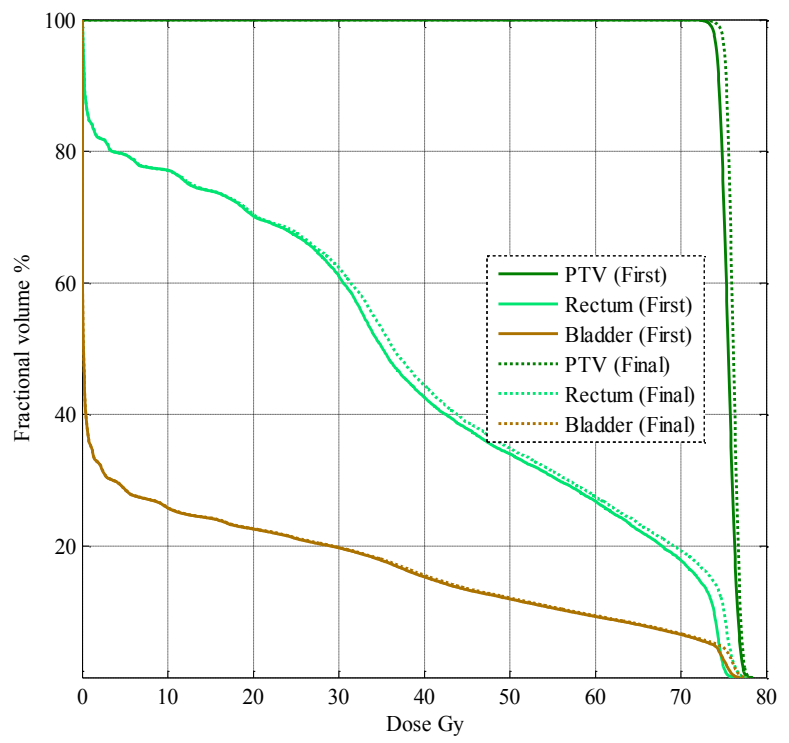

Figure 4. DVHs comparison for patient 1.

Next, the automatic plans generated by fuzzy FMO were compared with the manual plan reported in our published article ${ }^{[38][39]}$. Figure5.(a)-(c) show the comparative results regarding to PTV coverage, rectum sparing, and bladder sparing. The comparisons indicate that the automatic plans are better than that of manual plan. For example, the automatic plan for patient 10 in Figure5 yields better PTV coverage, better bladder-sparing and rectum-sparing compared with the manual plan. The same conclusions can be gained in other plan comparisons for testing cases except patient 6.The Wilcoxon matched-pair, signed-rank test using a significance level of 0.05 was performed for Figure5. However, no significant difference was found between them.

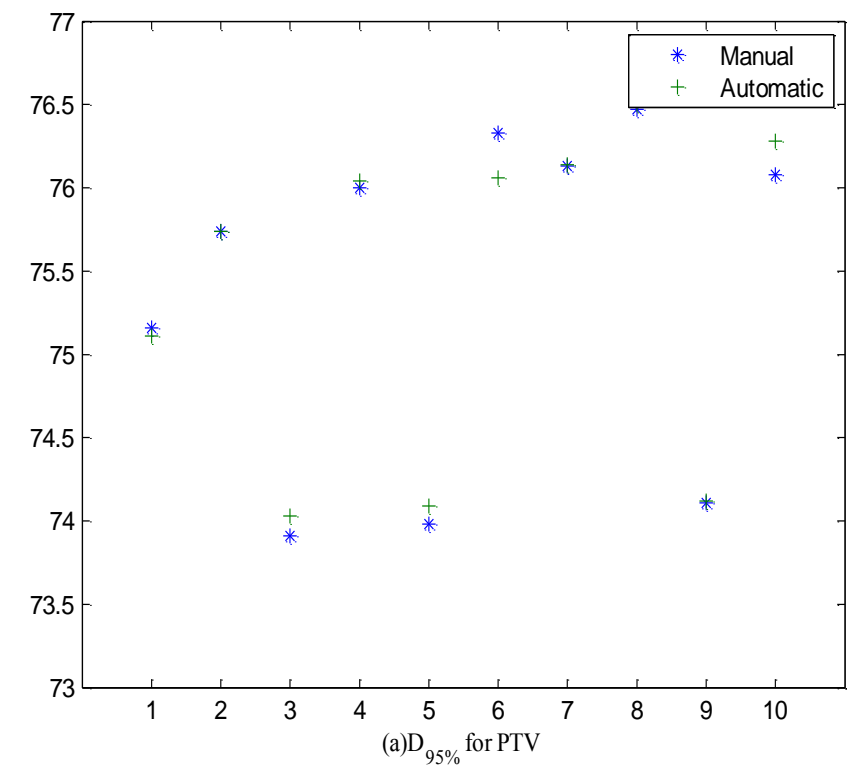



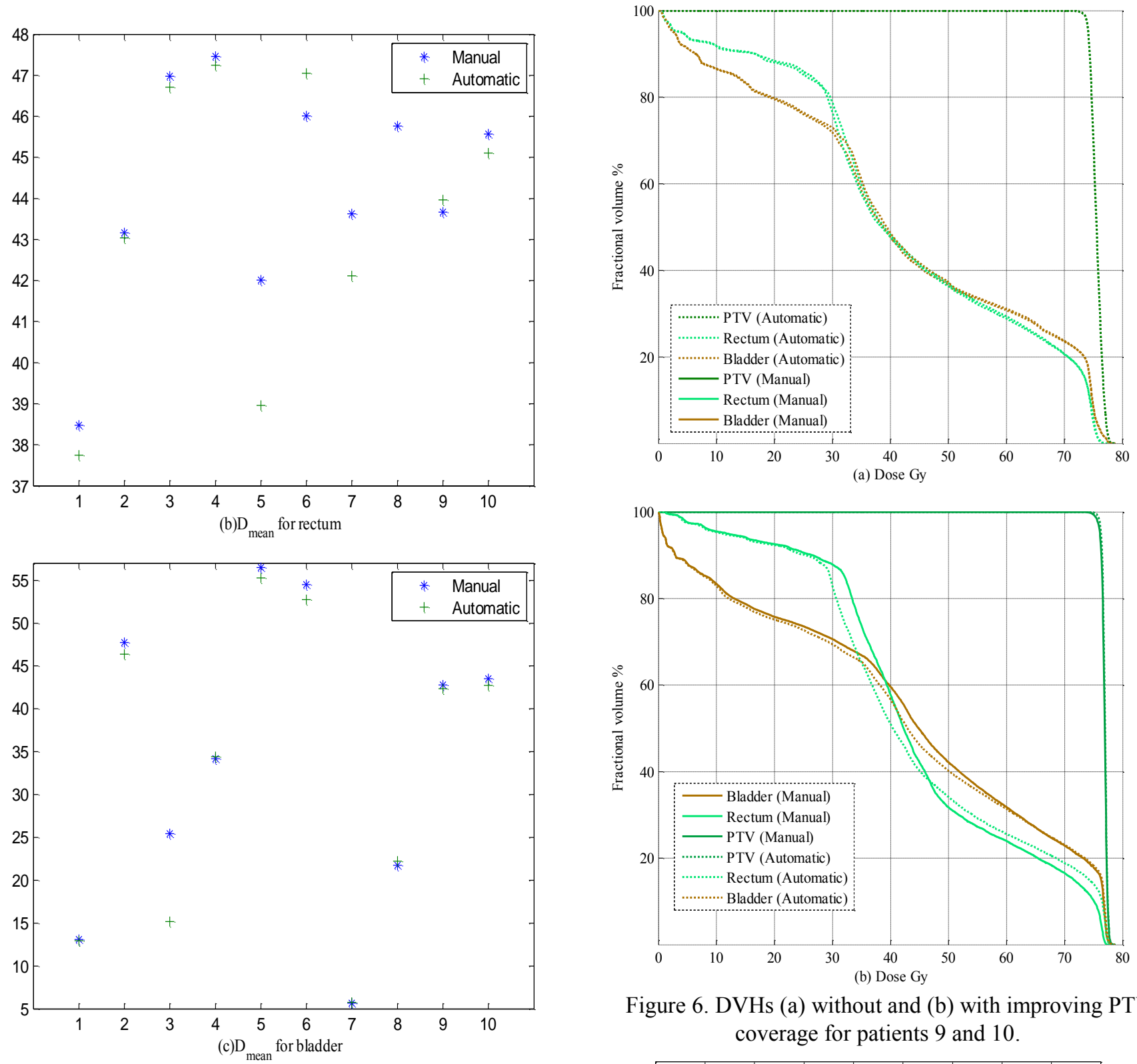

Figure 6. DVHs (a) without and (b) with improving PTV coverage for patients 9 and 10 .

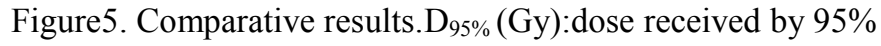
of the PTV volume; $D_{\text {mean }}(G y)$ : mean dose.

Two sets of comparative DVHs for two cases (patient 9 and patient 10) are shown in Figure 6. They are chosen as representatives with and without improving PTV coverage achieved by the fuzzy logic guided method compared to the manual FMO. In Figure6.(a),it is shown that the quality is similar between the automatic plan without improving PTV coverage and the manual plan. In Figure6.(b), the PTV coverage of the automatic plan with improving PTV coverage is better compared with the manual plan, while the high dose region of the rectum in automatic plan is worse than the manual plan because of the inherent contradiction of radiotherapy.

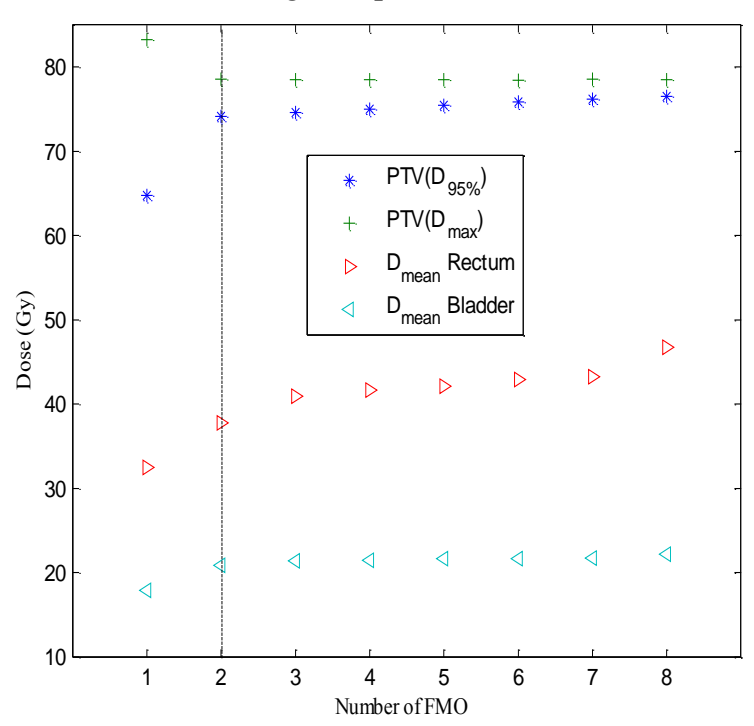

Figure 7. Dose change with the iteration number of FMO for patient 8 
To illustrate the way in which the automatic method tries to generate a desired plan, Figure 7 illustrate the dose change in the optimized structure with the iteration number of FMO for patient 8 . The vertical dotted line denotes the iteration where the first acceptable plan is found. We can see that by fuzzy logic guided changing objective function's parameters in an automatic fashion, an admissible plan can be found. After the first acceptable plan is reached, the algorithm tries to further improve the PTV coverage. Moreover, with the improvement of the PTV coverage, the dose deposited in OARs increases. It is not surprise because of the trade-off of radiotherapy.

\section{DISCUSSION AND CONCLUSION}

In this paper, we propose a fuzzy logic guided automatic method which does not need lengthy trial-and-error procedures for gEUD-based inverse treatment planning. The human planner's reasoning is imitated by the FIS in iterative optimization procedure. Some simple rules describe were used to update the parameters of optimization model. The efficiency of the automatic method was tested on 10 prostate cancer cases.Clinically accepted plans can be gained as shown in Table.

In our experiments, it takes 3 to 8 minutes for different prostate cases to produce an acceptable plan applying our proposed method, while for manual trial-and-error method, it needs experienced physicians to take 1 to 3 hours to obtained an acceptable plan. The substantial reduction in human intervention will improve the efficiency of radiotherapy greatly. With the increase of the number of sub-objective functions, the advantage of the proposed method becomes more and more apparent. The complexity of the patient anatomy and the number of loops executed by the algorithm will also influence the calculation time.

There persist challenges and room for improvement in this vein First, as to the selection of the input and output membership functions, the triangular and trapezoidal functions were chosen. We performed some experiments to investigate the influence of the shape and threshold values of membership functions on plan quality, experimental results show that they don't have effect on plan quality but have an effect on computational time and convergence. More efficient membership functions need to be further investigated. Second, in our work simple inference rules were used extracted from the experiences of the treatment planner. Although our adopted inference rules are suitable for all test cases, it should be noted that when more specific requirements are specified, the rule set will be more complex. To increase the versatility of the algorithm for different cases, we will introduce learning technique into radiotherapy optimization to automatically build FIS according to practical data.

Finally, the proposed fuzzy logic guided automatic optimization method can not only be applied in IMRT, but can also automatically generate volumetric modulated arc therapy plans. Related work is in progress.

\section{ACKNOWLEDGMENTS}

This work is supported by Program for the (Reserved)
Discipline Leaders of Taiyuan Institute of Technology [Y201701], Scientific and Technological Innovation Programs of Higher Education Institutions in Shanxi [2019L0924] .We would like to thank the anonymous reviewers for their valuable suggestions and comments which improved the quality of this paper greatly.

\section{REFERENCES}

[1] C. Shen, Y. Gonzalez, P. Klages, et al., "Intelligent Inverse Treatment Planning via Deep Reinforcement Learning, a Proof-of-Principle Study in High Dose-rate Brachytherapy for Cervical Cancer", Physics in Medicine and Biology, vol.64, pp.1-19, 2018.

[2] R. P. Li and F. F. Yin, "Optimization of inverse treatment planning using a fuzzy weight function', Medical Physics, vol. 27,pp. 691-70, 2000.

[3] H .Yan, F. F.Yin, H.Q. Guan and J. H.Kim, "Fuzzy logic guided inverse treatment planning", Medical. Physics, vol.30, pp. 2675-85, 2003.

[4] H.Yan, F.F.Yin, and C.Willett, "Evaluation of an artificial intelligence guided inverse planning system: Clinical case study", Radiotherapy and Oncology, vol.83, pp.76-85, 2007.

[5] F. Stieler, H. Yan, F. Lohr, F.Wenz and FF.Yin, "Development of a neuro-fuzzy technique for automated parameter optimization of inverse treatment planning", Radiation Oncology, vol.4, pp. 39, 2009. [6] J.Dias, H.Rocha, T.Ventura, B.Ferreira, and M. D. C.Lopes, "Automated fluence map optimization based on fuzzy inference systems”, Medical Physics, vol.43, pp.1083-1095, 2016.

[7] L.Y.Zhang, P.C.Zhang, Z.G. Gui,et al., "Plan optimization method of IMRT based on biological indices", Application Research of Computers, vol.34,pp.1303-1307,2017.

[8] S.Das, "A role for biological optimization within the current treatment planning paradigm", Medical Physics, vol.36, pp. 4672-4682, 2009.

[9] C.P. Guo, H.Z. Shu, Z.G. Gui, et al., "Improvement based on regularization for building hybrid criteria model of radiotherapy planning",Computer Engineering and Applications, vol. 53, pp. 23-28, 2017.

[10] Q.Wu, R.Mohan, A.Niemierko and R. Schmidtullrich, "Optimization of intensity-modulated radiotherapy plans based on the equivalent uniform dose", International Journal of Radiation Oncology Biology Physics, vol.52, pp. 224-235, 2002.

[11] C.Thieke, T.Bortfeld, A.Niemieko and S.Nill, "From physical dose constraints to equivalent uniform dose constraints in inverse radiotherapy planning", Medical Physics, vol.30, pp. 2332-2339, 2003.

[12] M.Hartmann and L.Bogner, "Investigation of intensity-modulated radiotherapy optimization with gEUD-based objectives by means of simulated annealing", Medical Physics, vol.35, pp. 2041-2049, 2008.

[13] X.S. Qi, V.A. Semenenko and X.A. Li, "Improved critical structure sparing with biologically based IMRT optimization", Medical Physics, vol.36, pp. 1790-1799, 2009.

[14] D.N.Mihailidis, B. Plants, L. Farinash, et al., "Superiority of equivalent uniform dose (EUD)-based optimization for breast and chest wall", Medical dosimetry,vol.35, pp. 67-76, 2010.

[15] C.Holdsworth, M.Kim, J.Liao and M.H. Phillips, "A hierarchical evolutionary algorithm for multiobjective optimization in IMRT", Medical Physics, vol.37, pp. 4986-4997, 2010. 
[16] T.Dirscherl, J.Alvarez-Moret and L.Bogner, "Advantage of biological over physical optimization in prostate cancer?", Zeitschrift Fur Medizinische Physik, vol.21, pp. 228-235, 2011.

[17] Q.Diot, B.Kavanagh, R.Timmerman and M.Miften, "Biological-based optimization and volumetric modulated arc therapy delivery for stereotactic body radiation therapy", Medical Physics, vol.39, pp. 237-245, 2012.

[18] T.F. Lee, H.M. Ting, P.J. Chao, et al., "Dosimetric advantages of generalised equivalent uniform dose-based optimisation on dose-volume objectives in intensity-modulated radiotherapy planning for bilateral breast cancer", The British Journal of Radiology, vol.85, pp. 1499-1506, 2012.

[19] RaySearch Laboratories AB SS, "Biological optimization using the equivalent uniform dose (EUD) in Pinnacle3", RaySearch White Paper, 2003.

[20] J.O. Deasy, A. I. Blanco and V. H. Clark, "CERR: a computational environment for radiotherapy research", Medical Physics, vol.30, pp. 979, 2003,

[21] Q . Wu and R. Mohan, "Algorithms and functionality of an intensity modulated radiotherapy optimization system", Medical Physics, vol.27, pp.701-711, 2000.

[22] A. Niemierko, "A generalized concept of equivalent uniform dose", Medical Physics, vol.26, pp. 1100 (abstract), 1999.

[23] L.A. Zahed, "Fuzzy sets", Information and Control, vol.8, pp. 338-353, 1965.

[24] L.A. Zahed, "The concept of a linguistic variable and its application to approximate reasoning", Information Sciences, vol.8, pp.199-249, 1975.

[25] L.A. Zahed, "Fuzzy logic", Computer, vol.1, pp. 83-93, 1988.

[26] L.A. Zahed, "Knowledge representation in fuzzy logic", IEEE Transactions on Knowledge and Data Engineering vol.1, pp. 89-100, 1989.

[27] J.Kumar, V.Kumar, K.Rana,"Control of Complex Systems Using Self Organizing Fuzzy Controller", Advances in Chaos Theory and Intelligent Control, vol.337,pp.753-772,2016.

[28] A.Mahmoudi, S.Sadi-Nezhad, A.Makui, "A Hybrid Fuzzy-Intelligent System for Group Multi-Attribute Decision Making", International Journal of Fuzzy Systems, vol.18, pp. 1117-1130, 2016.

[29] Z.Elouedi,N. B. Amor, "Special issue: Selected papers from Fuzzy Logic and Applications", Fuzzy Sets and Systems, vol. 366, pp.1-2,2019.

[30] S. Hrehova ,J. Mizakova J, "Evaluation a Process using Fuzzy Principles and Tools of Matlab",Int. J. of Applied Mathematics, ,vol.1, pp. 61-65,2019.

[31] H. Shah, M.Gopal, "A Reinforcement Learning Algorithm With Evolving Fuzzy Neural Networks", International Journal of Electrical Engineering and Computer Science (EEACS), vol.2, pp. 68-72,2020.

[32] L.B. Marks, E.D. Yorke and A. Jackson, "Use of normal tissue complication probability models in the clinic", International Journal of Radiation Oncology Biology Physics, vol. 76, pp. S10-S19, 2010.

[33] C.P.Guo, P.C.Zhang, Z.G.Gu iand H.Z.Shu, "An efficient method for improving the dose-volume-based optimization plan quality", IEEE access, vol.5, pp. 7520-7531, 2017.

[34] S.G.Nash, J. Nocedal, "A Numerical Study of the Limited Memory BFGS Method and the Truncated-Newton for Large Scale Optimization",Siam Journal on Optimization, vol.1,pp. 358-372,2016. [35] B.Emami, J .Lyman, A. Brown, et al, "Tolerance of normal tissue to therapeutic irradiation", International Journal of Radiation Oncology Biology Physics, vol.21, pp. 109-122, 1991.

[36] S.T.Peeters, M.S.Hoogeman, W.D.Heemsbergen,et al., "Rectal bleeding, fecal incontinence, and high stool frequency after conformal radiotherapy for prostate cancer: normal tissue complication probability modeling", International Journal of Radiation Oncology Biology Physics, vol.66, pp. 11-19, 2006.

[37] X.Wang, "Effectiveness of noncoplanar IMRT planning using a parallelized multiresolution beam angle optimization method for paranasal sinus carcinoma", International Journal of Radiation Oncology Biology Physics, vol.63,no.2,pp. 594-601,2005.

[38] C.P. Guo, H.Z. Shu, Z.G. Gui, L.Y. Zhang, "IMRT plan optimization based on hybrid criteria", Chinese Journal of biomedical engineering, vol. 35, pp. 712-718, 2016.

[39] C.P.Guo, P.C.Zhang, L.Y.Zhang, Z.G.Gui, and H.Z.Shu, "Application of optimization model with piecewise penalty to intensity-modulated radiation therapy", Future Generation Computer Systems, vol.81, pp. 280-290, 2018.

\section{Creative Commons Attribution License 4.0 (Attribution 4.0 International, CC BY 4.0)}

This article is published under the terms of the Creative Commons Attribution License 4.0 https://creativecommons.org/licenses/by/4.0/deed.en_US 